\section{Suchtkranke in der somatischen Medizin}

Zusammenfassung: Vor dem Hintergrund, dass Tabakrauchen und Alkoholkonsum zwei der größten vermeidbaren Gesundheitsrisiken sind, besteht das Ziel des Beitrages in der Darstellung der Häufigkeit der entsprechenden Probleme, der Vorbedingungen von adäquaten Interventionen und der Interventionen selber. Bisherige Studien zeigen u.a. 8,5\% Alkoholabhängige unter Patientinnen sowie $19,8 \%$ unter Patienten eines Allgemeinkrankenhauses, die in der Regel nicht wegen der Suchtmittelproblematik stationär aufgenommen worden waren. Gleichzeitig wird deutlich, dass die bisherige Gesundheitsversorgung diesen Problemen, ebenso wie bei dem Tabakrauchen, nicht gerecht wird. Voraussetzung adäquater Interventionen in der medizinischen Versorgung ist ein proaktiver Ansatz, der ein aktives Zugehen auf den Patienten ermöglicht. Als Grundlage hat sich das Transtheoretische Modell bewährt, das die Änderungsmotivation der Patienten individuell einschätzen und eine Indikation für die entsprechende Fokussierung der Beratung stellen lässt. Eine Reihe von Kurzinterventionen ist auf dieser Basis sinnvoll. Besonders bewährt und praktikabel ist die Motivierende Gesprächsführung. Es wird u.a. der Schluss gezogen, dass geeignete Verfahren der Frühintervention in neuen Feldern für die Suchtkrankenversorgung mittlerweile vorliegen und dass es jetzt auf die Implementierung in die Praxis ankommt.

Schlüsselwörter: Alkoholabhängigkeit - Rauchen - Medizinische Versorgung - Kurzintervention - Transtheoretisches Model

Addicted Patients in Somatic Medicine: Tobacco smoking and alcohol drinking are two of the major preventable health risks. Viewing this evidence, the aim of the present paper is to describe the prevalence of the respective behaviors in medical settings, the prerequisites for interventions and available techniques. Findings show that $8.5 \%$ of female and $19.8 \%$ of male inpatients admitted to one general hospital mostly not because of addictive behaviors - were alcohol dependent.

To date, for this group as well as for smokers health care services provide no adequate intervention addressing these behaviors. A prerequisite for adequate interventions is a proactive approach that directly contacts the patients. The

Suchttherapie Sonderheft 2001; 2: S15-S19

(C) Georg Thieme Verlag Stuttgart · New York ISSN 1439-9903
Ulrich John ${ }^{1}$, Ulfert Hapke ${ }^{1}$, Hans-jürgen Rumpf², Christian Meyer ${ }^{1}$, Gallus Bischof ${ }^{2}$

${ }^{1}$ Ernst-Moritz-Arndt-Universität Greifswald, Institut für Epidemiologie und Sozialmedizin, Suchtforschungszentrum

2 Medizinische Universität zu Lübeck, Klinik für Psychiatrie und Psychotherapie, Forschungsgruppe Substanzgebrauch: Therapie, Epidemiologie und Prävention

Transtheoretical Model can serve as a valuable framework for tailoring brief interventions according to individuals' motivation to change. An especially approved and practical method within this frame is Motivational Interviewing. It is concluded that suitable techniques for early intervention in medical settings exist. The next necessary step is to implement these approaches into daily routine.

Key words: Alcohol Dependence - Smoking - Medical Care - Brief Intervention - Transtheoretical Model

\section{Problemlage}

Tabakrauchen und gesundheitsschädlicher Alkoholkonsum bilden zwei der größten vermeidbaren Gesundheitsrisiken [1]. Allein Tabakrauchen ist ein Ursachenfaktor für 17\% aller Todesfälle während eines Jahres in Deutschland [2]. Es liegt auf der Hand, dass Suchtkrankenversorgung sich diesen besonders verbreiteten Problemen prioritär widmen muss. Tatsächlich aber steht in Deutschland das Minimum an Aktivitäten in Prävention und Therapie im Widerspruch zu den hochprävalenten begleitenden Problemen. Dieser Widerspruch besteht, obwohl neuere Forschung zeigt, dass wir über wirksame Interventionen verfügen. Sie sind jedoch nicht hinreichend bekannt. Das Ziel dieses Beitrages besteht darin, die Häufigkeit der Probleme, die Vorbedingungen für Interventionen und die Interventionen in der somatischen Medizin selber darzustellen. Dabei begrenzen wir uns auf die Substanzen, deren Konsum zu besonders vielen Gesundheitsstörungen beiträgt: Tabakrauchen und schädlicher Alkoholkonsum. Über psychotrope Medikamente ist der Informationsstand völlig unbefriedigend.

\section{Häufigkeit}

Für das Tabakrauchen sind mehr als 371 Erkrankungen oder Todesursachen bekannt, für die ein erhöhtes attributables Risiko besteht [2]. Bei riskantem Alkoholkonsum handelt es sich um 250 Erkrankungen (ICD-9 vierstellig) und zusätzlich ca. 80 Unfälle oder Verletzungen. Für Tabakrauchen ist bei dem überwiegenden Teil der Erkrankungs- oder Todesursachengruppen ein ursächlicher Zusammenhang belegt [3]. Tabakrauchen hat von allen gesundheitsrelevanten Verhaltensweisen den höchsten Anteil an den Todesursachen [1]. Riskanter Alkoholkonsum (20 g Reinalkohol pro Tag bei Frauen, $30 \mathrm{~g}$ pro Tag bei Männern) [4,5] trägt zu etwa einem Fünftel aller Todesfälle bei [6]. Das legt nahe, dass Raucher 
und riskante Alkoholkonsumenten in der somatischen Medizin überrepräsentiert sind. Um das zu prüfen, sind zwei Wege beschreitbar: Erstens lässt sich untersuchen, in wie vielen medizinischen Fachgebieten Patienten mit Substanzmissbrauch in ambulanter und stationärer Versorgung zu behandeln sind. Zweitens lassen sich die Häufigkeiten betroffener Patienten schätzen. Zur ersten Frage zeigt die Forschung, dass Alkoholmissbraucher und alkoholabhängige Patienten sich in allen medizinischen Fachgebieten der stationären Behandlung befinden $[7,8]$. Besonders relevant sind in Allgemeinkrankenhäusern die Abteilungen für Chirurgie und Innere Medizin, weil sie zwei Drittel der Behandlungsbetten stellen [7].

Ältere Studien in Deutschland waren zu wenig aufschlussreich, um Aussagen über Krankenhäuser oder Regionen zuzulassen. So wurden in mehreren Untersuchungen lediglich einzelne Krankenhausbereiche einbezogen. Die Prävalenzen der Alkoholabhängigkeit können aber zwischen einzelnen Abteilungen von Krankenhäusern sehr schwanken [9]. Darüber hinaus ergeben sich nicht unerhebliche Schwankungen über die Monate eines Jahres. Aus den Mängeln unzureichender Informationen in Deutschland heraus führten wir eine Untersuchung durch, die repräsentativ für ein Krankenhaus mit internistischer und chirurgischer Abteilung ist. Die Stichprobe umfasst 1309 Patienten, die nach stationärer Aufnahme gebeten wurden, Screening-Fragebogen zu Alkoholmissbrauch oder -abhängigkeit auf den Stationen auszufüllen. Weiterhin wurden die Behandlungsdaten der Patienten für Screening-Zwecke herangezogen. Ergab sich aus diesen oder aus dem Fragebogen-Screening ein positives Resultat, erhielt der Patient ein standardisiertes diagnostisches Interview (Schedules for Clinical Assessment in Neuropsychiatry [10]) zur Erstellung von Diagnosen gemäß ICD-10 und DSM-III-R. Nach Abschluss der Diagnostik erhielten die Patienten ein Beratungsgespräch, das die individuelle Änderungsbereitschaft berücksichtigte [11-16]. Die systematische Diagnostik in zwei Schritten führte zur Bestimmung der Patienten als Alkoholabhängige, Alkoholmissbraucher, Patienten mit Verdachtsdiagnose oder Patienten ohne alkoholbezogene Diagnose. Eine Verdachtsdiagnose wurde vergeben, wenn weder die Kriterien für einen Alkoholmissbrauch noch für eine Alkoholabhängigkeit gemäß ICD-10 oder DSM-III-R erfüllt, aber wenn Hinweise auf eine Alkoholproblematik aufgrund von Aufzeichnungen in der Krankenakte oder aufgrund der Laborergebnisse vorhanden waren. Von den Patientinnen im Alter von 18 bis 64 Jahren wiesen 7,0\% eine gegenwärtige, 1,5\% eine remittierte Alkoholabhängigkeit, 1,3\% einen Alkoholmissbrauch und 3,8\% eine Verdachtsdiagnose auf. Von den Patienten zeigten 16,5\% eine gegenwärtige Alkoholabhängigkeit, 3,3\% eine remittierte Alkoholabhängigkeit, 7,2\% einen Alkoholmissbrauch und $13,6 \%$ eine Verdachtsdiagnose [7]. In dem Einzugsgebiet des Krankenhauses fanden sich aufgrund einer repräsentativen Bevölkerungsuntersuchung 1,3\% aktual Alkoholabhängige, weitere $1,1 \%$ Alkoholmissbraucher und weitere 5,1\% Risikokonsumenten (mindestens 20 g Reinalkohol pro Tag bei Frauen und mindestens $30 \mathrm{~g}$ pro Tag bei Männern; vgl. [17]). Die Raucherraten in dem Krankenhaus betrugen bei den 18- bis 64-jährigen Patientinnen, die vier Tage pro Woche oder öfter rauchten, 34,7\% und bei den Patienten 49,8\% im Vergleich zu 35,3\% der Frauen und $\mathrm{zu}$ 39,3\% der Männer im Alter von 18 bis 64 in der Bevölkerung, die derzeit täglich Zigaretten rauchten.
Eine Analyse der Behandlungsakten ergab, dass zwei Drittel der Patienten sich im Krankenhaus wegen Erkrankungen befanden, die aufgrund der Literatur als Folgen des Alkoholkonsums bekannt sind [18]. Insgesamt aber weisen Alkoholabhängige eine große Zahl unterschiedlicher Behandlungsdiagnosen auf. Unter den älteren Patienten (ab 65 Jahren) sind die Diagnosen einer Alkoholabhängigkeit oder eines Alkoholmissbrauches deutlich seltener (3,5\% Alkoholabhängigkeit oder -missbrauch, $2 \%$ remittierte Alkoholabhängigkeit [19]). In der Ambulanz des untersuchten Krankenhauses wurden über mehrere 24-stündige Behandlungsphasen hinweg die Patienten hinsichtlich einer möglichen Alkoholproblematik mit Screening-Verfahren untersucht: 33,8\% der Patienten fielen durch eine positive Screening-Diagnose auf [20].

In einer weiteren Studie zur Prävalenz von alkoholbezogenen Störungen ergab sich in einer Zufallsauswahl von Allgemeinarztpraxen (929 Screening-Untersuchungen mit Patienten im Alter von 14 bis 75 Jahren) bei $12,1 \%$ der männlichen Patienten eine gegenwärtige Alkoholabhängigkeit und bei weiteren 5,6\% ein Alkoholmissbrauch. Von den Frauen waren es 4,2\% mit gegenwärtiger Alkoholabhängigkeit und 2,1\% mit Alkoholmissbrauch [21].

Die Erfahrung im Umgang mit den Patienten, die eine alkoholbezogene Diagnose aufwiesen, zeigt, dass unter den vorgefundenen Versorgungsbedingungen zu den Suchtmittelproblemen auf Station von dem behandelnden und pflegenden Personal nicht hinreichend beraten oder anderweitig interveniert werden konnte. Eine Untersuchung zu dieser Problematik belegt gleichzeitig, dass mittels eines systematischen Screenings mit nachfolgender Intervention durch Suchtberater, die werktags auf Station anwesend sind (Liaisondienst), eher Aufgaben der Frühintervention übernommen werden können als durch ein Konsiliarsystem, in dem der behandelnde Arzt im Falle von substanzbezogenen Störungen einen Suchtberater rufen kann [22]. Im Liaisondienst führen die Suchtberater das systematische Screening bei jedem Patienten durch, im Falle eines positiven Ergebnisses auch die Diagnostik auf Störungen im Zusammenhang mit dem Konsum psychotroper Substanzen. Bei jeder positiven Diagnose gehen sie aktiv mit einer geeigneten Intervention auf den Patienten zu. Das ist ein proaktiver Ansatz, der auf den ersten Blick besonders aufwändig erscheint. Aufgrund der Erfahrungen ist aber zu erwarten, dass der Personaleinsatz erstens zu einer wesentlichen Verbesserung der Gesundheitsversorgung und zweitens zu einer Reduktion der Inanspruchnahme medizinischer Leistungen bei den betroffenen Patienten führt.

Zu Tabakrauchen sowie Alkoholabhängigkeit und -missbrauch bei Patienten im Allgemeinkrankenhaus lässt sich das Fazit ziehen, dass es sich um häufige Probleme handelt, denen jedoch nicht adäquat begegnet wird. Aufgrund der Daten lässt sich hochrechnen, dass von allen sich in stationärer Behandlung befindlichen Alkoholabhängigen während eines Jahres in Deutschland 91\% im Allgemeinkrankenhaus, weitere 6\% im psychiatrischen Krankenhaus und 3\% in den Fachkrankenhäusern für Entwöhnungsbehandlungen behandelt werden [7]. Bei Störungen durch Tabakrauchen zeigt sich eine ähnliche Problematik wie bei den Alkoholstörungen. 


\section{Vorbedingungen für Interventionen}

Bei suchtmittelbezogenen Störungen sind als zwei grundlegende Voraussetzungen für Interventionen die Änderungsbereitschaft und die bisherige Inanspruchnahme von Hilfen zu betrachten. Gemäß diesen Voraussetzungen sind dann spezifische Interventionen einzusetzen. Eine weitere Voraussetzung für Interventionen ist eine effiziente Entdeckung durch Screening- und diagnostische Verfahren [23,24].

Entscheidend für den Einsatz von Interventionen ist, dass auch Personen mit geringer Änderungsmotivation erreicht werden. Einen geeigneten Ansatz dazu bildet das Transtheoretische Modell nach Prochaska \& DiClemente [25]. Es hat die Änderung im Verhalten auch bei solchen Personen zum Ziel, die noch nicht änderungsbereit sind. Im Transtheoretischen Modell werden dementsprechend Stadien der Änderungsabsicht differenziert: Im Stadium der Absichtslosigkeit bestehen keine Wünsche, das Konsumverhalten zu ändern. Das Stadium der Absichtsbildung ist vor allem durch ein Anwachsen der Auseinandersetzung mit dem eigenen Trinkverhalten und seinen Konsequenzen charakterisiert. Die Person wägt Argumente für eine Änderung des Konsumverhaltens mit Argumenten $\mathrm{ab}$, die dagegen sprechen. Im Stadium der Vorbereitung werden Aktivitäten geplant, im Stadium der Handlung konkrete Schritte unternommen, z. B. kauft sich der Raucher ein Nikotinpflaster. Im Stadium der Aufrechterhaltung wird das Zielverhalten, z.B. die Abstinenz, aufrechterhalten. Die Prozesse oder Strategien der Änderung bilden die entscheidenden Merkmale für das Voranschreiten durch die Stadien, das auch das Ziel entsprechender motivationaler Interventionen ist. $\mathrm{Zu}$ unterscheiden sind als kognitiv-affektive Strategien das Steigern des Problembewusstseins, emotionales Erleben, Neubewertung der persönlichen Umwelt, Selbstneubewertung, die Wahrnehmung förderlicher Umweltbedingungen und als verhaltensorientierte Strategien Selbstverpflichtung, Kontrolle der Umwelt, Gegenkonditionierung, das Nutzen hilfreicher persönlicher Beziehungen und die Belohnung von Verhaltensweisen [25]. Das Modell ist übertragbar auf unterschiedliche Verhaltensweisen im $\mathrm{Zu}$ sammenhang mit der Änderung des Substanzgebrauches, Aktivitäten, die zur Abstinenz führen können, oder die Abstinenz selber. Das Transtheoretische Modell erlaubt eine Fülle von Maßnahmen der Frühintervention, um einer regional bedarfsgerechten Suchtkrankenversorgung näher zu kommen [26].

Eine besonders geeignete Interventionsform ist die Motivierende Gesprächsführung [27-29]. Sie wurde im Kontext der Behandlung von substanzabhängigen Menschen entwickelt. Ziel ist die Förderung der Motivation, an einem

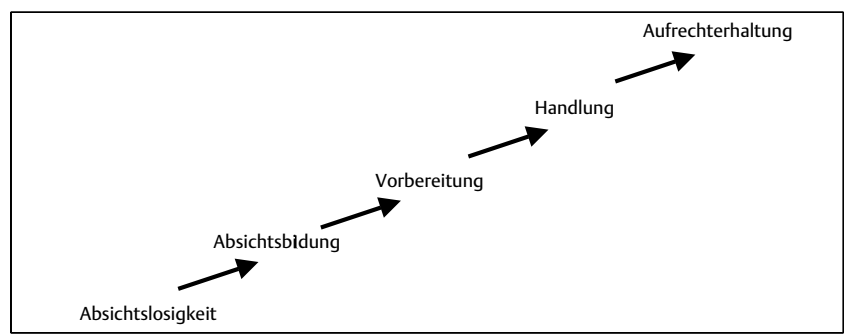

Abb. 1 Stadien der Änderungsabsicht.
Substanzkonsum und den resultierenden Problemen eine Änderung in Richtung einer Problemlösung zu realisieren. Sie baut auf Grundsätzen der humanistischen Psychotherapieschulen auf, ist jedoch direktiver und basiert auf mehreren Konzepten und Methoden. Beratungs- und Behandlungsstrategien sind spezifisch auf unterschiedliche psychologische Prozesse abgestimmt, die im jeweiligen Stadium der Änderungsbereitschaft Verhaltens- und Einstellungsänderungen fördern können. Wichtig für ein Setting mit einer motivational niedrigen Zugangsschwelle zur Beratungssituation ist der Fokus des Konzeptes: Es eignet sich besonders für Betroffene, die keine Änderungsbereitschaft oder Ambivalenz zeigen. Die Autoren der Motivierenden Gesprächsführung schlagen fünf Grundprinzipien vor, fünf allgemeine Techniken, strukturierte Vorgehensweisen und spezifische Strategien im Umgang mit Widerstand. Die fünf Grundprinzipien der Motivierenden Gesprächsführung lauten: 1. eine empathische Grundhaltung des Beraters, die es dem Klienten erleichtert, seine Zurückhaltung aufzugeben und sich zu öffnen, 2. Förderung der Wahrnehmung von Diskrepanzen zwischen Zielen und Wünschen des Klienten und seinem Substanzkonsum, 3. Vermeidung von konfrontativen, moralisierenden und stigmatisierenden Argumentationen, 4. Verständnis von Abwehr als gestörte Interaktion zwischen Klienten und Berater, die es zu bearbeiten gilt, 5. die Erfahrung von Selbstwirksamkeit als ein wichtiges Element von erfolgreicher intentionaler Verhaltensänderung, die der Berater durch seine Intervention vermitteln soll. Fünf allgemeine Techniken umfassen: 1. offene Fragen stellen, nicht Fragen, die nur mit Ja oder Nein beantwortbar sind, 2. reflektierendes Zuhören, 3. Bestätigen und Unterstützen des Patienten, 4. Zusammenfassen eines Gesprächsblockes, 5. Äußerungen zur Eigenmotivation des Patienten herausarbeiten. Strukturierte Vorgehensweisen umfassen z.B. die Schilderung eines typischen Alltages oder die Exploration von Vergangenheit, Gegenwart und Zukunft des Patienten. Die Strategien im Umgang mit Widerstand sollen bewusst eine Konfrontation vermeiden helfen. $\mathrm{Zu}$ ihnen gehören z.B. die Widerspiegelung von Ambivalenz bei dem Patienten oder die Betonung seiner persönlichen Entscheidungsfreiheit [15]. Die Motivierende Gesprächsführung ist im Allgemeinkrankenhaus sehr praktikabel. So wurde sie auf unterschiedliche Stadien der Änderungsabsicht unter Bedingungen des Allgemeinkrankenhauses abgestimmt [16]. Wenig aufwändige Beratungen haben sich auch im Vergleich zu stationären Motivationstherapien im psychiatrischen Krankenhaus als sehr ernst zu nehmende Ansätze erwiesen [30]. Zusätzliche Kurzinterventionsansätze über die Beratung hinaus sind in einer gesonderten Übersicht [31] dargestellt.

Schließlich ist als Voraussetzung für die Intervention eine adäquate und kostensparende Entdeckung bei den zu erwartenden hohen Fallzahlen bedeutend. Dazu wurde als im internationalen Vergleich besonders viele Fälle entdeckendes, aber gleichzeitig zeitlich sehr wenig aufwändiges Instrument der Lübecker Alkoholabhängigkeits- und -missbrauchs-Screening-Test entwickelt [23]. Ist nach einem Screening die Diagnose gemäß den Klassifikationssystemen ICD-10 oder DSM-IV bestätigt, lässt sich auch die Schwere der Abhängigkeit gemäß dem Abhängigkeitssyndrom erfassen [24]. Darüber hinaus gibt es Verfahren, um die Probleme, die eine Alkoholabhängigkeit begleiten oder ausformen, zu erfassen z.B. mit dem ASI [32]. 


\section{Intervention}

Ein wesentliches Ziel von Frühinterventionen ist die Inanspruchnahme von Angeboten der Suchtkrankenversorgung. Einer Untersuchung der Erwachsenenbevölkerung einer Region in Norddeutschland zufolge hatten 70,9\% gegenwärtig Alkoholabhängige keinerlei Kontakt zur formellen Suchtkrankenhilfe, 14,5\% zeigten eine geringfügige Inanspruchnahme, z.B. weniger als 10 Selbsthilfegruppenbesuche, weitere $14,5 \%$ wiesen eine darüber hinausgehende Inanspruchnahme auf, z. B. mindestens 10 Selbsthilfegruppenbesuche oder viermaliges Aufsuchen einer Suchtberatungsstelle oder einer Behandlung, die über den körperlichen Entzug von Alkohol hinausging. Andererseits hatten $80,0 \%$ der Alkoholabhängigen mindestens einmal im Jahr einen praktischen Arzt oder einen Arzt für Allgemeinmedizin konsultiert [33]. Zu berücksichtigen ist in diesem Zusammenhang, dass die Wege aus der Sucht sehr heterogen sind und keine einfachen Klassifizierungen differenzieller Therapie- oder Interventionsindikation ermöglichen, die voraussagen lassen, ob die Betroffenen die Remission allein oder mit einer speziellen professionellen Hilfe realisieren können [34-37].

Raucher zeigen eine unbedeutende Inanspruchnahme von therapeutischen Hilfen (Entwöhnungsbehandlung oder Hilfsmittel wie Nikotinpflaster). In der Bevölkerungsstudie der 4075 untersuchten Erwachsenen gaben lediglich 8\% der Raucher an, Entwöhnungsverfahren, wie Entwöhnungskurse oder Akupunktur, in Anspruch genommen zu haben [38].

Die als Alkoholabhängige entdeckten Patienten aus der dargestellten Allgemeinkrankenhausstudie erhielten gemäß der Motivierenden Gesprächsführung eine Beratung, die in der Regel deutlich unter einer Stunde lag. Das Ziel war, die Patienten zu motivieren, weitere Hilfe in Anspruch zu nehmen, sei es eine Selbsthilfegruppe, sei es eine Beratungsstelle oder Behandlung. In einer Untersuchung von 187 so beratenen Patienten zeigte sich im Laufe des ersten Jahres nach der stationären Behandlung eine Steigerung der Inanspruchnahme von Hilfen von 10,5\% Teilnahme an Selbsthilfegruppen vor dem Krankenhausaufenthalt auf 27,2\% nach dem Krankenhausaufenthalt, bei Suchtberatung von 8,8 auf $37,7 \%$, bei Entzugsbehandlung von 18,4 auf 33,3\%, bei Entwöhnungsbehandlung von 2,6 auf $12,3 \%$ [7,16]. In einer weiteren Studie wurden die an der Motivierenden Gesprächsführung orientierte Motivierungsarbeit in drei Einzelberatungen verglichen mit einer 14-tägigen Motivationstherapie. Die Beratungsgruppe unterschied sich im Laufe der ersten sechs Monate nach Behandlung von der Motivationstherapiegruppe nicht in der Rate der abstinenten ehemaligen Patienten [30].

Insgesamt erweist sich die im Einzelfall wenig aufwändige Frühintervention, die Stadien der Änderungsabsicht aus dem Transtheoretischen Modell und die die Motivierende Gesprächsführung nutzt, aufgrund der Erfahrung als sehr praktikabel und geeignet. Strukturell sollte ein sekundärpräventives Programm aufgestellt werden, das sich nach den bisherigen Erfahrungen am besten mit einem Liaisondienst von Suchtberatern im Allgemeinkrankenhaus durchführen lässt.

\section{Schlussfolgerungen}

Die Suchtkrankenversorgung erweist sich bei bevölkerungsbezogener Betrachtung als unzureichend. Gleichzeitig liegen aber geeignete Interventionsformen, die bei den zu erwartenden hohen Fallzahlen sehr zeitsparend sein müssen, vor. Im Einzelnen lassen die wissenschaftlichen Resultate und die Erfahrungen diese Schlüsse ziehen: 1. Die Suchtkrankenversorgung in Deutschland hat bei einem hohen Standard im therapeutischen Bereich die sehr großen Gruppen Abhängiger unter den Patienten in der somatischen Basisversorgung der Bevölkerung zu sehr außer Acht gelassen. Gleichzeitig liegen aber praktikable Interventionsansätze vor. Proaktive Interventionen, die in einem ersten Schritt mittels Screening-Fragebögen Patienten mit Alkoholproblemen identifizieren lassen, um anschließend durch einen Liaisondienst Interventionen durchzuführen, erwiesen sich als ökonomisch und praktikabel. Demzufolge sind in der Suchtkrankenversorgung die Verlagerung von Aktivitäten oder zusätzliche Aktivitäten in diesen Bereichen sinnvoll. 2. Die betroffenen Patienten nehmen die vorhandenen Behandlungsangebote in deutlich zu geringem Maße an. Verfahren, die das Versorgungsangebot ergänzen können und dem Bedarf sowie den individuellen Voraussetzungen besser gerecht werden, sind in ersten Ansätzen entwickelt. Besonders eignen sich Verfahren, die an den Stadien der Änderungsabsicht orientiert sind. Es kommt jetzt auf die Implementierung in die Praxis an. 3. Das Ziel muss eine bevölkerungsbezogene Suchtkrankenversorgung sein, die suchtmittelbezogene Gesundheitsstörungen mit den größten Häufigkeiten in den Fokus rückt.

\section{Danksagung}

In dieser Arbeit genannte eigene Forschungsarbeiten wurden gefördert durch das Bundesministerium für Bildung und Forschung, das Bundesministerium für Gesundheit sowie das Sozialministerium des Landes Mecklenburg-Vorpommern.

\section{Literatur}

${ }^{1}$ McGinnis JM, Foege WH. Mortality and morbidity attributable to use of addictive substances in the United States. Proceedings of the Association of American Physicians 1999; 111: 109-118

${ }^{2}$ John U, Hanke M. Tabakrauch-attributable Mortalität in den deutschen Bundesländern. Das Gesundheitswesen 2001; 63: 363-369

${ }^{3}$ Doll R. Risk from tobacco and potentials for health gain. International Journal Tuberc and Lung Disease 1999; 3: 90-99

${ }^{4}$ British Medical Association. Guidelines on sensible drinking. British Medical Association. London, 1995

${ }^{5}$ Bühringer G, Augustin R, Bergmann E, Bloomfield K, Funk W, Junge $B$ et al. Alkoholkonsum und alkoholbezogene Störungen in Deutschland. Baden-Baden: Nomos, 2000

${ }^{6}$ John U, Hanke M. Tobacco- and alcohol-related mortality. Greifswald: Universität Greifswald, Institut für Epidemiologie und Sozialmedizin, 2001

${ }^{7}$ John U, Hapke U, Rumpf HJ, Hill A, Dilling H. Prävalenz und Sekundärprävention von Alkoholmissbrauch und -abhängigkeit in der medizinischen Versorgung. Baden-Baden: Nomos, 1996

${ }^{8}$ Moore RD, Bone LR, Geller G, Mamon JA, Stokes EJ, Levine DM. Prevalence, detection, and treatment of alcoholism in hospitalized patients. Journal of the American Medical Association (JAMA) 1989; 261: 403-407 
${ }^{9}$ John U, Rumpf HJ, Hapke U. Estimating prevalence of alcohol abuse and dependence in one general hospital - an approach to reduce sample selection bias. Alcohol and Alcoholism 1999; 34: 786-794

${ }^{10}$ Gülick-Bailer , Maurer K, Häfner H. Schedules for Clinical Assessment in Neuropsychiatry (SCAN). German version. Bern: Huber, 1995

${ }^{11}$ Hapke U, Rumpf HJ, John U. Alkoholabhängigkeit und -missbrauch im Allgemeinkrankenhaus - Förderung der Inanspruchnahme suchtspezifischer Versorgungsangebote. In: John U. Deutsche Hauptstelle gegen die Suchtgefahren (Hrsg). Regionale Suchtkrankenversorgung. Konzepte und Kooperationen. Freiburg: Lambertus, 1997: 101-108

${ }^{12}$ Hapke U, Kremer G, Rumpf H-J. Motivational Interviewing. In: Stimmer, F. (Hrsg). Suchtlexikon. München: Oldenbourg, 2000: 410-413

${ }^{13}$ Hapke U, Rumpf HJ, Hill A, John U. Prävalenz und Sekundärprävention von Alkoholmissbrauch, Alkoholabhängigkeit und Alkoholfolgeerkrankungen in der medizinischen Basisversorgung. Schriftenreihe zur Suchtkrankenhilfe des Senators für Frauen, Gesundheit, Jugend, Soziales und Umweltschutz Referat Suchtkrankenhilfe. Bremen, 1998; Band 2

${ }^{14}$ Hapke U, Rumpf HJ, John U. Grundprinzipien motivierender Beratung. Schriftenreihe zur Suchtkrankenhilfe des Senators für Frauen, Gesundheit, Jugend, Soziales und Umweltschutz Referat Suchtkrankenhilfe. Bremen, 1998; Band 2

${ }^{15}$ Hapke U, Rumpf HJ, Schumann A, John U. Beratung von Menschen mit problematischem Alkohol-, Medikamenten- oder Drogenkonsum auf Basis des Transtheoretischen Modells. In: Keller S (Hrsg). Motivation zur Verhaltensänderung: das transtheoretische Modell in Forschung und Praxis. Freiburg, 1999: 81-91

${ }^{16}$ Hapke U. Sekundärpräventive Interventionen bei Patienten mit einer Alkoholproblematik im Allgemeinkrankenhaus: Theoretische Grundlagen und empirische Befunde. Freiburg: Lambertus, 2000

${ }^{17}$ Meyer C, Rumpf HJ, Hapke U, John U. Impact of DSM-IV psychiatric disorders: prevalence, persistence and life satisfaction in the general population of northern Germany. Greifswald: Universität Greifswald, Institut für Epidemiologie und Sozialmedizin, 2001

${ }^{18}$ Gerke P, Hapke U, Rumpf HJ, John U. Alcohol-related diseases in general hospital patients. Alcohol \& Alcoholism 1997; 32: 179-184

${ }^{19}$ Rumpf HJ, Bromisch B, Botzet M, Hill A, Hapke U, John U. Epidemiologie des Alkoholmissbrauchs im höheren Lebensalter. In: Havemann-Reinecke U, Weyerer S, Fleischmann $\mathrm{H}$ (Hrsg). Alkohol und Medikamente, Missbrauch und Abhängigkeit im Alter. Freiburg: Lambertus, 1998: 29-37

${ }^{20}$ Polte M, Hapke U, Rumpf HJ, John U. Schätzung der Prävalenz von Alkoholmissbrauch und -abhängigkeit in der Notfallambulanz eines Allgemeinkrankenhauses. Sucht 1999; 45: 187-194

${ }^{21}$ Hill A, Rumpf HJ, Hapke U, Driessen M, John U. Prevalence of alcohol dependence and abuse in general practice in Germany a representative study. Alcoholism: Clinical and Experimental Research 1998; 2: 935-940

${ }^{22}$ Hapke U, Rumpf HJ, John U. Differences between hospital patients with alcohol problems referred for counselling by physicianś routine clinical practice versus screening questionnaires. Addiction 1998; 93: 208-209

${ }^{23}$ Rumpf HJ, Hapke U, John U. Der Lübecker Alkoholabhängigkeitsund -missbrauchs-Screening-Test (LAST). Manual. Göttingen: Hogrefe, 2001

${ }^{24}$ John U, Hapke U, Rumpf HJ. Skala zur Erfassung der Schwere einer Alkoholabhängigkeit, SESA. Göttingen: Hogrefe, in Druck
${ }^{25}$ Keller S, Velicer WF, Prochaska JO. Das Transtheoretische Modell Eine Übersicht. In: Keller S (Hrsg). Motivation zur Verhaltensänderung: Das Transtheoretische Modell in Forschung und Praxis. Freiburg: Lambertus, 1999: 17-44

${ }^{26}$ John U, Hapke U, Rumpf HJ, Meyer C, Schumann A, Bischof G Das TTM als Instrument in der gesundheitlichen Versorgung der Bevölkerung - der Ansatz der Community Medicine. In: Keller S (Hrsg). Motivation zur Verhaltensänderung - Das Transtheoretische Modell in Forschung und Praxis. Freiburg: Lambertus, 1999: 45-55

${ }^{27}$ Hapke U, Rumpf HJ, John U. Beratung von alkoholabhängigen Patienten im Allgemeinkrankenhaus. In: Deutsche Hauptstelle gegen die Suchtgefahren (Hrsg). Alkohol, Konsum und Missbrauch. Alkoholismus, Therapie und Hilfe. Freiburg: Lambertus, 1996: 345-354

${ }^{28}$ Hapke U, Rumpf HJ, Hill A, John U. Alkoholprobleme in der medizinischen Basisversorgung - Prävalenz und sekundärpräventive Strategien. In: Aktion Psychisch Kranke (Hrsg). Innovative Behandlungsstrategien bei Alkoholproblemen. Freiburg: Lambertus, 1997: 49-59

${ }^{29}$ Miller WR, Rollnick S. Motivierende Gesprächsführung. Freiburg: Lambertus, 1999

${ }^{30}$ John U, Veltrup C, Driessen M, Wetterling T, Dilling H. Motivationsarbeit mit Alkoholabhängigen. Freiburg: Lambertus, 2000

${ }^{31}$ Rumpf H-J, Hapke U, Meyer C, Bischof G, John U. Kurzinterventionen bei alkoholbezogenen Störungen. In: Deutsche Hauptstelle gegen die Suchtgefahren (Hrsg). Individuelle Hilfen für Suchtkranke - Früh erkennen, professionell handeln, effektiv integrieren. Freiburg: Lambertus, 2000: 211-219

${ }^{32}$ Gsellhofer B, Küfner H, Vogt M, Weiler D. European Addiction Severity Index. EuropASI. Deutsche Bearbeitung. Baltmannsweiler: Schneider, 1999

${ }^{33}$ Rumpf HJ, Meyer C, Hapke U, Bischof G, John U. Inanspruchnahme suchtspezifischer Hilfen bei Alkoholabhängigen und -missbrauchern: Ergebnisse der TACOS-Bevölkerungsstudie. Sucht 2000; 46: 9-17

${ }^{34}$ Bischof G, Rumpf HJ, Hapke U, Meyer C, John U. Gender differences in natural recovery from alcohol dependence. Journal of Studies on Alcohol 2000; 61: 783-786

${ }^{35}$ Bischof G, Rumpf HJ, Hapke U, Meyer C, John U. Maintenance factors of recovery from alcohol dependence in treated and untreated individuals. Alcoholism: Clinical and Experimental Research 2000; 24: 1773-1777

${ }^{36}$ Bischof G, Rumpf HJ, Hapke U, Meyer C, John U. Remission ohne formelle Hilfen und Inanspruchnahme stationärer Behandlung bei Alkoholabhängigen: Ein Vergleich auslösender Faktoren. Sucht 2000; 46: 54-61

${ }^{37}$ Rumpf HJ, Bischof G, Hapke U, Meyer C, John U. Studies on natural recovery from alcohol dependence: Sample selection bias by media solicitation? Addiction 2000; 95: 765-775

${ }^{38}$ Meyer C, Rumpf HJ, Hapke U, John U. Inanspruchnahme von Hilfen zur Erlangung der Nikotin-Abstinenz. Sucht 2000; 46: 398-407

\section{Prof. Dr. Ulrich John}

Direktor des Institutes für Epidemiologie und Sozialmedizin Ernst-Moritz-Arndt Universität

Walther-Rathenau-Straße 48

17487 Greifswald

E-mail: ujohn@uni-greifswald.de 